\title{
Biosensors: the new wave in cancer diagnosis
}

This article was published in the following Dove Press journal:

Nanotechnology, Science and Applications

24 December 2010

Number of times this article has been viewed

\section{Brian Bohunicky' Shaker A Mousa ${ }^{1,2}$ \\ 'The Pharmaceutical Research Institute at Albany College of Pharmacy and Health Sciences, Rensselaer, NY, USA; ${ }^{2}$ College of Medicine, King Saud University, Riyadh, Saudi Arabia}

Correspondence: Shaker A Mousa The Pharmaceutical Research Institute at Albany College of Pharmacy and Health Sciences, One Discovery Drive, Rensselaer, NY 12144, USA

Tel +I 5186947397

Fax + I 5186947567

Emailshaker.mousa@acphs.edu

\begin{abstract}
The earlier cancer can be detected, the better the chance of a cure. Currently, many cancers are diagnosed only after they have metastasized throughout the body. Effective, accurate methods of cancer detection and clinical diagnosis are urgently needed. Biosensors are devices that are designed to detect a specific biological analyte by essentially converting a biological entity (ie, protein, DNA, RNA) into an electrical signal that can be detected and analyzed. The use of biosensors in cancer detection and monitoring holds vast potential. Biosensors can be designed to detect emerging cancer biomarkers and to determine drug effectiveness at various target sites. Biosensor technology has the potential to provide fast and accurate detection, reliable imaging of cancer cells, and monitoring of angiogenesis and cancer metastasis, and the ability to determine the effectiveness of anticancer chemotherapy agents. This review will briefly summarize the current obstacles to early detection of cancer and the expanding use of biosensors as a diagnostic tool, as well as some future applications of biosensor technology.
\end{abstract}

Keywords: biosensor, oncogene, nanotechnology, biotechnology, cancer detection, diagnosis, point-of-care

\section{Introduction}

Cancer is the second leading cause of death in the United States and is expected to claim the lives of nearly 570,000 people in 2010 (http://www.cancer.org/). This number equates to $\sim 1500$ deaths/day. One in two men will be diagnosed with some form of cancer over the next year, while for women, the probability of being diagnosed with cancer is just over 30\% ( 1 in 3). Cancer can take over 200 distinct forms, including lung, prostate, breast, ovarian, hematologic, skin, and colon cancer, and leukemia, and both environmental factors (eg, tobacco smoke, alcohol, radiation, and chemicals) and genetic factors (eg, inherited mutations and autoimmune dysfunction) are associated with an increased risk of developing cancer. Bacterial and viral infections are also strongly associated with some types of cancer (ie, stomach cancers and cervical cancer, respectively). The most common type of cancer in men and women is prostate and breast cancer, respectively, with 192,000 new cases of each reported annually. Although cancer is most commonly diagnosed later in life $(77 \%$ of cases are diagnosed in individuals aged 55 years and older), $\sim 11,000$ cases will be diagnosed in children aged 0-14 years. Not only is cancer deadly, but it is one of the most expensive diseases in the United States, with total cancer-related expenses in 2008 of approximately $\$ 228.1$ billion (see http://www.cancer.org/). submit your manuscript $\mid$ www.dovepress.com

Dovepress

DOI: $10.2147 /$ NSA.S1 3465
Nanotechnology, Science and Applications 20I I:4 I-10

(C) 20II Bohunicky and Mousa, publisher and licensee Dove Medical Press Ltd. This is an Open Access article which permits unrestricted noncommercial use, provided the original work is properly cited. 
The American Cancer Society (ACS) estimates that the average 5-year survival rate for all cancers for the years 19962004 has increased to $66 \%$ (up from $50 \%$ for $1975-1977$ ). This increase in survival can be attributed to technological advances resulting in better treatment and improvement in early diagnosis. However, the 5-year survival for certain cancers such as liver, pancreatic, and lung remains very low $(6 \%-16 \%)$. The use of emerging biosensor technology could be instrumental in early cancer detection and more effective treatments, particularly for those cancers that are typically diagnosed at late stages and respond poorly to treatment, resulting in improvements in patient quality of life and overall chance of survival.

Cancer is defined as abnormal and uncontrolled cell growth due to an accumulation of specific genetic and epigenetic defects, both environmental and hereditary in origin. Unregulated cell growth leads to the formation of a tumor mass that over time becomes independent of normal homeostatic checks and balances. Tumor cells in essence become resistant to apoptosis and other antigrowth defenses within the body. ${ }^{1}$ As the cancer progresses, the tumor begins to spread beyond the site of origin and metastasize to other body organs and systems, at which point, the cancer is essentially incurable.

The two major tumorigenesis mechanisms are activation of oncogenes and inactivation of tumor suppressor genes (TSGs). ${ }^{2-5}$ Activation of oncogenes occurs through mutation or duplication of a normal gene (a proto-oncogene) involved in the regulation of cell growth, proliferation, and/or differentiation. This typically results in constitutive activation or excess levels of a normal gene product, leading to the deregulation of cell growth, increased cell division, and tumor formation. Perhaps more so than any other type of oncogene, growth factor receptors have been investigated as potential cancer biomarkers. The human epidermal growth factor receptor Her-2, for example, is amplified in $\sim 33 \%$ of all breast cancers, and cancers with amplified Her- 2 tend to grow and spread more aggressively. Thus, knowledge of Her-2 status is critical in determining the proper course of treatment. Trastuzumab, a recombinant humanized monoclonal antibody directed against Her-2 as targeted therapy for breast cancer, is now standard adjuvant treatment for patients with this type of amplified gene expression. ${ }^{6,7}$

TSGs are involved in the regulation of inappropriate cell growth and proliferation by slowing or stopping cell division. Three of the most well-studied TSGs in cancer are retinoblastoma protein $(\mathrm{Rb}), \mathrm{BRCA} 1 / 2$, and $\mathrm{p} 53 .{ }^{3}$ The $\mathrm{Rb}$ is a master regulator of cell division, and mutation of Rb plays a role in many cancers. Point mutations and deletions are the most common causes of inactivation of the $\mathrm{Rb} 1$ gene. $^{8-10}$ BRCA1 is a DNA repair enzyme, which is involved in 'proofreading' newly replicated DNA for fidelity and presence of mutations. DNA repair enzymes normally function to remove replication errors before the cell divides. BCRA1 gene mutations account for about $50 \%$ of hereditary breast cancers and $80 \%-90 \%$ of hereditary breast and ovarian cancers. ${ }^{11,12}$ Finally, the p53 protein is a master regulator of apoptosis or programmed cell death. Mutations in p53 are found in brain, breast, colon, lung, hepatocellular carcinomas, and leukemia. Another major concern with the loss of p53 is that it can serve as a mechanism of chemotherapeutic drug resistance. ${ }^{4,5,13}$ The development of biosensors that can detect the presence of mutations in $\mathrm{p} 53, \mathrm{Rb}$, and BRCA1 is of great importance in terms of being better able to determine cancer susceptibility as well as more accurate prognosis and treatment regimens.

\section{Cancer biomarkers}

The National Cancer Institute (NCI) defines a biomarker as "a biological molecule found in blood, other body fluids, or tissues that is a sign of a normal or abnormal process or of a condition or disease. A biomarker may be used to see how well the body responds to a treatment for a disease or condition"..$^{14}$ Biomarkers can be of various molecular origins, including DNA (ie, specific mutation, translocation, amplification, and loss of heterozygosity), RNA, or protein (ie, hormone, antibody, oncogene, or tumor suppressor). Cancer biomarkers are potentially one of the most valuable tools for early cancer detection, accurate pretreatment staging, determining the response of cancer to chemotherapy treatment, and monitoring disease progression. ${ }^{15,16}$ Biomarkers are typically detected in human fluids such as blood, serum, urine, or cerebral spinal fluid, but they can also be present in or on tumor cells. ${ }^{17}$ A partial list of tumor biomarkers is presented in Table 1. Most of these biomarkers, however, have yet to demonstrate sufficient sensitivity and specificity for translation into routine clinical use or for treatment monitoring. This is an area that biosensor technology can potentially improve upon. Discussion of some of the major cancer biomarkers is presented below.

\section{Prostate-specific antigen}

Prostate-specific antigen (PSA) was one of the first tumor biomarkers to be identified and put into routine clinical use 
Table I Common biomarkers utilized for cancer detection 17,18,22

\begin{tabular}{ll}
\hline Type of cancer & Biomarker \\
\hline Breast & BRCAI, BRCA2, CA I5-3, CA I25, CA 27.29, CEA, \\
& NY-BR-I, ING-I, HER2/NEU, ER/PR \\
Colon & CEA, EGF, P53 \\
Esophageal & SCC \\
Liver & AFP, CEA \\
Lung & CEA, CA I9-9, SCC, NSE, NY-ESO-I \\
Melanoma & Tyrosinase, NY-ESO-I \\
Ovarian & CA I25, HCG, P53, CEA, CA 549, CASA, CA 19-9, \\
& CA I5-3, MCA, MOV-I, TAG72 \\
Prostate & PSA \\
\hline
\end{tabular}

for screening and diagnosis of prostate cancer. Studies have shown that above-normal PSA levels correlate directly with prostate cancer. A normal level of PSA is $4.0 \mathrm{ng} / \mathrm{mL}$. A study conducted by Smith found that roughly $30 \%$ of men with a PSA level between 4.1 and $9.9 \mathrm{ng} / \mathrm{mL}$ had prostate cancer. ${ }^{18}$ In addition to prostate cancer, elevated PSA levels may also indicate benign prostatic hyperplasia, prostatitis (inflammation of the prostate), or smaller tumors that do not prove to be fatal. Thus, PSA levels are not always indicative of malignant tumors, a fact that has prompted considerable controversy about the value of routine PSA screening for prostate cancer. Small tumors that are detected by PSA screening may in fact grow so slowly that death from the tumor would not be possible in a man's life span. Furthermore, treatment of these slow-growing tumors is costly and often involves lifechanging surgeries that may not be necessary. However, in a study of 695 men with prostate cancer who were assigned to either radical prostatectomy or observation (watchful waiting), it was shown that prostatectomy reduced morbidity, mortality, the risk of metastasis, and local progression more than watchful waiting, ${ }^{19}$ suggesting that treatment of any kind is more valuable than simple observation. Thus, despite the controversy surrounding PSA screening, it may be less harmful for a patient to receive unnecessary treatment for a benign condition than to not be treated for a malignant tumor.

Another issue with the PSA test is that false positives are common, and many men with elevated PSA levels do not have prostate cancer at all. ${ }^{20,21}$ This tendency with PSA screening is an area that biosensors can help by eliminating some of the ambiguity surrounding the common screening methods used today.

\section{Cancer antigen 125}

Elevated cancer antigen (CA) 125 is most commonly associated with ovarian cancer and is also linked to cancers of the uterus, cervix, pancreas, liver, colon, breast, lung, and digestive tract. Several nonpathological conditions such as menstruation and pregnancy can also result in increased levels of CA $125 .{ }^{22}$ CA 125 is elevated in $90 \%$ of women with advanced ovarian cancer and in $40 \%$ of patients with intra-abdominal malignancy. However, it should also be noted that in Stage 1 ovarian cancer, $50 \%$ of patients will have normal CA 125 levels. ${ }^{22,23}$ Other biomarkers that are linked to ovarian cancer are germ cell in origin such as human chorionic gonadotrophin (HCG), alpha-fetoprotein (AFP), and lactate dehydrogenase (LDH). ${ }^{24}$ Elevated CA 125 following total abdominal hysterectomy or bilateral salpingooophorectomy, which may follow first-line chemotherapy treatment, is suggestive of disease recurrence or treatment failure. In addition, increases in CA 125 have been used to identify the progression of benign cells to malignant cells. ${ }^{25,26}$ Overall, CA 125 is a valuable biomarker not only for cancer diagnosis, but also for various other aspects of cancer treatment and progression.

\section{CA 15-3}

CA 15-3 is an important biomarker analyzed in breast cancer patients. Other biomarkers that are linked to breast cancer are carcinoembryonic antigen (CEA), BRCA1, BRCA2, and CA $27.29 \cdot{ }^{17,27} \mathrm{CA} 15-3$ is used clinically most often to monitor patient therapy in cases of advanced breast cancer. In patients with breast cancer, it has been shown that CA 15-3 concentrations increase by $10 \%$ in Stage I cancer, $20 \%$ in Stage II, $40 \%$ in Stage III, and $75 \%$ in Stage IV breast cancer. ${ }^{16}$ Tampellini and colleagues investigated the relationship between CA 15-3 levels and breast cancer and showed that individuals with values of $<30 \mathrm{U} / \mathrm{mL}$ before treatment onset had significantly higher survival times than individuals who had higher levels. The study also found that higher CA 15-3 values correlated with extensive metastasis. ${ }^{28}$ Another study determined that rising CA 15-3 values after treatment can indicate disease recurrence. ${ }^{29}$ At present, CA 15-3 levels are considered along with tumor size, cancer stage, and negative risk factors (ie, Her-2 and ER/PR status) in determining treatment protocols. Other conditions that can cause CA 15-3 levels to increase are endometriosis, pelvic inflammatory disease, hepatitis, pregnancy, and lactation.

\section{Cancer-testis antigens}

Cancer-testis (CT) antigens are a unique class of cancer biomarker. They are highly expressed in many tumors, but not in normal cells, except for germ cells of the testis. Thus, they have been heavily pursued as potential immunogenic targets for cancer immunotherapies (ie, cancer vaccines), and 
autoantibodies to CT antigens have been pursued as cancer biomarkers. New York esophageal squamous cell carcinoma 1 (NY-ESO-1), encoded by $C T A G-1 B$, is a highly immunogenic member of this class of antigens, eliciting strong humoral and cellular immune responses in a high percentage of NYESO-1 positive cancers. The titer of antibodies to NY-ESO-1 has been shown to correlate with disease progression, as well as with disease response/resection. ${ }^{30}$ One of the advantages of CT autoantibodies as cancer biomarkers is that they are present in serum, which is far easier to obtain than tissue biopsies, and are very stable proteins. They may, therefore, be of particular importance in predicting cancer occurrence and/or progression. One of the disadvantages of CT antigens is that they are rarely tumor-specific, and many tumors will express more than one CT antigen. Multiplex biosensors from which one can derive a CT antigen profile have the potential to greatly improve the use of CT antigens in cancer detection, diagnosis, and prognosis.

\section{RCASI (EBAG-9)}

Receptor-binding cancer antigen expressed on SiSo cells (RCAS1) has been shown to be overexpressed in nearly $100 \%(98.4 \%)$ of gastric carcinomas and correlates closely with gastric tumor progression. ${ }^{31}$ RCAS1 has also been implicated as a marker of poor prognosis in gallbladder, esophageal, breast, and endometrial cancer and with cancer relapse in laryngeal and pharyngeal cancer. ${ }^{32}$ Thus, in terms of cancer biomarkers, RCAS1 expression appears to be highly indicative of cancer progression and perhaps prognosis. As with most of the cancer biomarkers discussed here, RCAS is overexpressed by many different types of tumors and should be factored in with other parameters when establishing treatment regimens.

\section{Proteomics and new cancer biomarkers}

The convergence of three major areas of research — advances in proteomic technologies (ie, LC-MS/MS, MALDI-MS), sequencing of the human genome, and the development of sophisticated software algorithms for comparing and analyzing large data sets derived from MS data-has resulted in rapid progress in the identification of new cancer biomarkers. The power of combining these techniques is that proteomic profiles can be generated using tumor tissue biopsies or plasma proteins as the source material. Current MS techniques can generate hundreds of thousands of individual mass spectra from a given protein sample. Publically available software tools can sort these spectra and compare the sequences to each other and to annotated genome databases to enable quantitative comparative assessment of proteomes from different sources (ie, tumor and nearby normal tissue). In this way, proteins that are overexpressed or downregulated in a tumor sample can be identified as putative cancer biomarkers. Readers are referred to several recent reviews on the field of proteomics and tumor biomarkers. ${ }^{33-36}$

\section{Biosensors and cancer}

A biosensor is a device used to detect a biological analyte, be it environmental or biological in origin (ie, within the human body). Information such as whether or not the analyte is present and at what level is transduced into an electrical signal that can be amplified, displayed, and analyzed. Examples of analytes include proteins (antigen, antibody, and enzyme), nucleic acid, or other biological or metabolic component (ie, glucose). In medicine, biosensors can be used to monitor blood glucose levels in diabetics, detect pathogens, and diagnose and monitor cancer. Environmental applications of biosensors include the detection of harmful bacteria or pesticides in air, water, or food (ie, during food preparation). The military has a strong interest in the development of biosensors as counter bioterrorism devices that can detect elements of chemical and biological warfare to avoid potential exposure or infection. The vision of the future for biosensors even includes chip-scale devices placed on the human body for monitoring vital signs, correcting abnormalities, or even signaling a call for help in an emergency. In theory, the applications of biosensors are limitless.

In terms of cancer, the analyte being detected by the biosensor is a tumor biomarker, examples of which were presented earlier in the review. Thus, by measuring levels of certain proteins expressed and/or secreted by tumor cells, biosensors can detect whether a tumor is present, whether it is benign or cancerous, and whether treatment has been effective in reducing or eliminating cancerous cells. Biosensors that can detect multiple analytes may prove particularly useful in cancer diagnosis and monitoring, since most types of cancer involve multiple biomarkers. The ability of a biosensor to test for multiple markers at once not only helps with diagnosis, but also saves time and financial resources. ${ }^{17}$

A biosensor is made up of three components: a recognition element, a signal transducer, and a signal processor that relays and displays the results. The molecular recognition component detects a 'signal' from the environment in the form of an analyte, and the transducer then converts the biological signal to an electrical output. ${ }^{37}$ The basic structure and function of a biosensor is shown in Figure 1. 


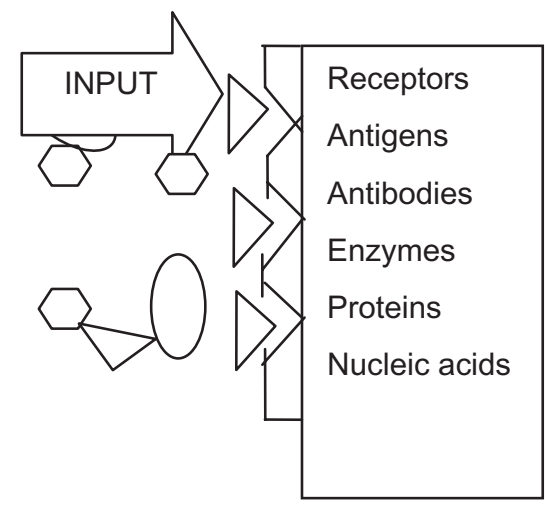

Sample analyte
Recognition

element

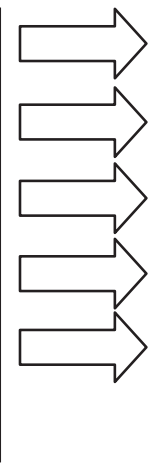

Electrochemical-potentiometric, amperometric

Optical-luminescence, fluorescence, colorimetry

Mass change-piezoelectric, acoustic wave

Calorimetric - thermal

Transducer

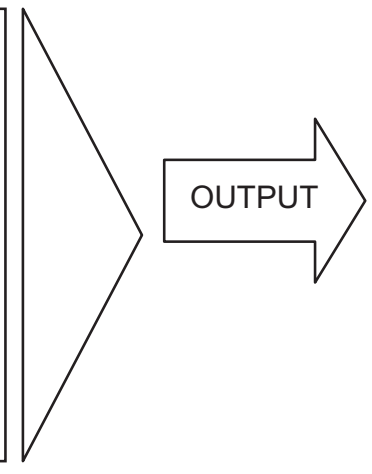

Amplifier data processing

Figure I Illustration depicting the process by which biosensors function.

\section{Biosensor recognition element}

The recognition element is a critical component of the biosensor. Early biosensors used naturally occurring recognition elements that were purified from biological or environment systems. With advances in technology and synthetic chemistry, many biosensor recognition elements used today are synthesized in the laboratory to allow for improved stability and reproducibility of biosensor function. Examples of recognition elements are receptor proteins, antigens, antibodies, enzymes, and nucleic acids.

\section{Receptor recognition elements}

Cell surface receptors are targets for drug delivery and are useful for monitoring the effectiveness of cancer therapeutics. Activation or inactivation of receptor molecules triggers diverse signaling events within a cell. Altering the function of a receptor can result in opening or closing of ion channels and changes to membrane permeability, activation of adenyl cyclase and second messengers, and activation of small $\mathrm{G}$ proteins, kinases, phosphatases, and transcription factors. However, despite the fact that receptor activity governs numerous aspects of cell behavior and receptor proteins would be valuable as biosensor recognition elements, their use in biosensors is problematic. Receptors function properly only in the milieu of a bilayer lipid membrane, making it difficult to obtain purified receptor proteins in high yield that are stable and that retain sufficient activity. ${ }^{38}$

\section{Antigen/antibody recognition elements}

Antigen- and antibody-based recognition elements are among the most rapid detection systems. A critical benefit of this type of element is the inherent specificity of antibody-antigen interactions and the fact that the target molecule does not need to be purified before recognition. The recognition element for PSA biosensors, some of the most widely used biosensors in clinical use for the detection of prostate cancer, is an anti-PSA antibody. Anti-PSA recognition elements have been linked to microcantilever-based transducers and to surface plasmon resonance-based sensors, in which changes in vibrational frequency upon antigen binding to antibody are used to detect PSA. ${ }^{39,40}$

\section{Enzymes}

Allosteric enzymes show great potential as recognition elements. In most cases, the regulatory subunit takes on the role of the recognition element and the catalytic site becomes the transducer. ${ }^{41}$ One of the most advanced sensors of this class, and in general, is the glucose sensor, which uses glucose oxidase as the recognition element. Glucose oxidase catalyzes the oxidation of glucose in the presence of oxygen to yield gluconolactone and hydrogen peroxide. Typically, an amperometric transducer will measure the rate of elimination of oxygen or the rate of formation of hydrogen peroxide and convert this into a glucose reading. ${ }^{38}$

\section{Nucleic acids}

Aptamers are oligonucleotides that are selected from among a pool of thousands of different sequences for very high binding affinities for their targets. As a result, they are rapidly emerging as biosensor recognition elements. A combinatorial chemistry-based technology termed SELEX (systematic evolution of ligands by exponential enrichment) has been developed to generate nucleic acid ligands from a library of DNA and RNA oligonucleotides. Because the number of 
potential ligands is very high and the molecules are selected for high affinity binding to a target ligand, the applications of SELEX are nearly unlimited. Biosensors built on this type of system have proven to be useful in the discovery of new biomarkers that are critical to early cancer diagnosis. Cancer biomarkers that have been discovered using SELEX technology include NY-BR-1 and ING-1, as well as the CT antigens CAGE-1 and NY-ESO-1. ${ }^{42-44}$ Aptamers have also been used in small sensing arrays for the detection of cancer proteins. Another characteristic of aptamers is that they contain allosteric properties. RNAzymes and DNAzymes are examples of allosteric aptamers. These compounds are very useful for monitoring disease and have shown promise in the areas of Alzheimer's disease and diabetes. ${ }^{38}$

\section{Biosensor transducer}

A molecular recognition element can be interfaced to a number of different signal transducers. The transducer converts the molecular signal into an electric or digital signal that can be quantified, displayed, and analyzed. Transducers fit into four general categories: electrochemical (ie, amperometric and potentiometric), optical (ie, colorimetric, fluorescent, luminescent, and interferometric), mass based (ie, piezoelectric and acoustic wave), and calorimetric (temperature based). ${ }^{45}$

\section{Electrochemical biosensors}

Electrochemical biosensors are the most common type of biosensor in use today due to their portability, cost effectiveness, small size, and ease of use. Electrochemical biosensors can be used at home or in the doctor's office as point-of-care (POC) devices. As mentioned above, the glucose sensor, which has revolutionized the way that blood glucose readings are taken and recorded, is an electrochemical biosensor. ${ }^{17}$ Potentiometric and amperometric biosensors are the two most common types of electrochemical biosensors. Potentiometric biosensors use ion-selective electrodes to detect an electrical response in the molecular recognition element. ${ }^{37}$ Not yet in clinical use, but showing great promise in the area of cancer detection, is a light-addressable potentiometric sensor (LAPS) that is coupled to a phage recognition element. ${ }^{46}$ The phage-LAPS was able to detect the cancer biomarker hPRL-3 and cancer cells (MDA/MB231 breast cancer cell line) with high sensitivity. This new potentiometric-based biosensor has been proposed for use in the clinical detection of cancer and anticancer drug evaluation.

Amperometric transducers measure current that is produced when a potential is placed between two electrodes.
Oxidation or reduction reactions produce a current, which can then be measured. ${ }^{47}$ Amperometric-based biosensors for cancer detection that utilize sequence-specific DNA as the recognition element have the potential to be extremely useful in cancer diagnosis. ${ }^{48,49}$ These sensors can detect the presence of gene mutations associated with cancer through recognition of and hybridization to specific DNA sequences present in the genomes of cancerous cells. A breakthrough using this type of technology was made by Wang and Kawde, ${ }^{50}$ who used chrono-potentiometric transduction biosensors to determine that mutations of BRCA1 and BRCA2 are associated with hereditary breast cancer.

The GlucoWatch ${ }^{\mathrm{TM}}$ is a glucose sensor that was developed to enable noninvasive, continuous measurement of blood glucose levels in diabetic patients. The GlucoWatch ${ }^{\mathrm{TM}}$ is worn like a wristwatch and takes blood glucose readings through the skin via reverse iontophoresis, a process whereby a small electrical signal brings glucose to the skin surface so that it can be measured. Glucose signals are converted into an electrical signal through an amperometric transducer. This type of device helps patients maintain their blood glucose readings without the pain associated with traditional injections. The GlucoWatch $^{\mathrm{TM}}$ also has an alarm to alert the patient when blood glucose readings are out of the normal range and a way to record multiple glucose readings. ${ }^{51,52}$

Electrochemical biosensors offer the capability of detecting damaged DNA as well as the carcinogens that caused the damage. ${ }^{47}$ Electrochemical transducer technology is also widely used in immunoassays and protein arrays. Immunosensors, in which an antibody is coupled to an electrochemical transducer, have also been used for measuring cancer biomarkers.

The realization of multiplex biosensors that are capable of detecting multiple CAs, thus providing more accurate diagnosis and monitoring, is a key advance in biosensor technology. This type of biosensor has to contain multiple transducers that are individually targeted to specific proteins or antigens for detection. Because the technology is reliable, low cost, and easily scaled-down with the use of semiconductor materials, electrochemical-based biosensors have been key players in the area of multiplex biosensors. Multifunctional antibody arrays based on ELISA methodology are the most popular multiplex devices used for cancer protein analysis. $^{17}$

Asphahani and Zhang ${ }^{53}$ demonstrated the feasibility of a cell-based electrochemical biosensor that measures changes in cell impedance in response to analyte. These cell-based biosensors are commonly referred to as cytosensors and 
use live cells as the biological sensing element in order to monitor changes induced by various stimuli. In terms of cancer therapy, this type of sensor can be used to monitor the effects of anticancer agents on their target molecules. Most anticancer drugs work by killing cancer cells via p53-mediated apoptosis. During apoptosis, the cell undergoes drastic changes in membrane integrity, ion channel permeability, and overall morphology. Cytosensors would be able to detect such cellular changes more rapidly and effectively than a sensor that uses a purified component (ie, receptor or enzyme), thus more accurately determining the pharmaceutical efficiency of the anticancer agent in question.

\section{Optical biosensors}

Optical biosensors are light-based sensors that measure changes in specific wavelengths of light. The transducer can be luminescence, fluorescence, colorimetric, or interferometric based. Optical transducers convert changes in wavelengths or SPR in response to analyte recognition into an electrical/digital readout. ${ }^{17}$ Photonic crystal biosensors are a newly emerging class of biosensors that use an optical transducer. The photonic crystal sensor is designed to capture light from very small areas or volumes, allowing for greater sensitivity of measurement, and then transmit that light into a high electromagnetic field for display. By measuring the light reflected by the crystal, this technique can detect when and where cells or molecules bind to or are removed from the crystal surface. Using this type of biosensor, Chan and colleagues were able to monitor changes in proliferation and apoptosis of breast cancer cells exposed to doxorubicin and determine the $\mathrm{IC}_{50}$ of doxorubicin. ${ }^{54}$ This type of biosensor technology would be useful for pretreatment screening of effective doses in order to balance efficacy of treatment and toxicity.

Another exciting example of this application of this type of technology to cancer detection is the esophageal laser fluorescence-based optical biosensor for the diagnosis and monitoring of cancers of the throat. After being swallowed by the patient, the device directs a laser beam emitting a specific wavelength of light onto the surface of the esophagus. The esophageal wall reflects light at very specific wavelengths, based on whether the tissue contains cancerous cells or normal cells. This sensor has been tested on over 200 patients and found to accurately diagnose cancer over $98 \%$ of the time. ${ }^{55}$ By using this type of biosensor, surgical biopsies and the pain and recovery time associated with them could be avoided.

\section{Mass-based biosensors}

Piezoelectric and acoustic wave biosensors make up the class of mass-based biosensors. In terms of cancer detection, piezoelectric biosensors are more commonly used. Piezoelectric sensors are based on changes in the mass of quartz crystals when potential energy is applied to them. This change in mass generates a frequency, which can be converted into a signal. Immunosensors and microcantilever sensors that use piezoelectric technology have proven useful in the identification of cancer biomarkers. ${ }^{17}$ A study conducted by Dell'Atti and colleagues ${ }^{56}$ illustrated the use of a piezoelectric biosensor coupled with PCR amplification to detect point mutations in the human p53 gene, which underlie in part almost all types of cancer. Because mutations of p53 are critical to cancer development and the effectiveness of cancer treatments, there has been considerable effort in the development of quick, inexpensive, and effective ways to detect p53 mutations.

\section{Calorimetric biosensors}

Calorimetric biosensors are less common than other biosensors for cancer diagnostics, but the introduction of nanotechnology to the field of biosensors has increased the range of applications for these types of biosensors. Calorimetric biosensors measure exothermic reactions. Many enzymatic reactions generate heat, and changes in heat can be used to measure analyte concentration. The reaction is monitored by measuring enthalpy changes, which indirectly provide information about substrate concentration. ${ }^{37}$ Calorimetric biosensors are not commonly used for the diagnosis and prognosis of cancer, but some cancerdetecting capabilities have been demonstrated. A recent report by Medley and colleagues ${ }^{57}$ demonstrated the use of an aptamer-based gold nanoparticle calorimetric biosensor for the detection of cancer. The researchers were able, using the gold nanoparticles, to differentiate two different cell types, acute leukemia cells and Burkitt's lymphoma cells. This report illustrates the feasibility of combining aptamerbased recognition elements with a calorimetric transducer to detect cancer cells ${ }^{57}$ and potentially discriminate between normal and cancer cells.

\section{Biosensors and nanotechnology}

Nanotechnology is a rapidly emerging field that is having an enormous impact on biosensors, and by extension, the diagnosis, prognosis, and monitoring of cancer. Most cancer is diagnosed only after it has metastasized, making it much more deadly and difficult to treat. Roughly $60 \%$ of cancer cases 
are diagnosed in patients after the tumor has metastasized. The application of nanotechnology to biosensor development improves the chances of detecting cancer earlier, thus improving patient survival rates. One example of this is in the diagnosis of cancer by magnetic resonance imaging (MRI), one of the most common imaging technologies in use today for cancer diagnosis and monitoring. A major drawback of MRI is that it cannot detect entities that are smaller than a few centimeters. The use of nanomaterials as imaging agents allows for more sensitive and precise measurement of cancerous tissues. Liposomes, dendrimers, buckyballs, and carbon nanotubes are all examples of nanomaterials that have been used to improve cancer imaging. ${ }^{58}$ Additionally, the use of nanotechnology means smaller sensors, which translates into better access to and detection of cancer markers, as well as more powerful and specific signal enhancements, reduced cost, and high throughput detection. ${ }^{1}$

Nanoparticles are defined as particles on the scale of $1-100 \mathrm{~nm}$ in diameter. The small size of nanoparticles allows for a greater surface to volume ratio. This increase in ratio allows for better detection, imaging, and prognosis methods and improved drug delivery to tumor sites that were previously not accessible. Nanocantilevers, nanowires, and nanochannels are all examples of structures that have been exploited for the detection of cancer-specific molecular events and improved signal transduction. ${ }^{59}$ Zhang and colleagues ${ }^{60}$ developed a nanowire-based biosensor for the detection of micro-RNAs (miRNAs). miRNAs are important regulators of gene expression and are associated with cancer development. Traditional methods of detecting miRNA such as northern blot analysis have low sensitivity and are time consuming and costly. The development of a sensitive, inexpensive, easy-to-use biosensor for detecting cancer-related miRNAs represents an advance in the use of miRNAs as cancer biomarkers.

The use of single-walled carbon nanotubes (SWCNTs) has greatly enhanced the detection capabilities of electrochemical biosensors, providing increased sensitivity to enzymatic reactions. SWCNTs have increased activity toward hydrogen peroxide and $\mathrm{NADH}^{61}$ and have been used in nucleic acid-based sensors and immunosensors for cancer biomarkers to enhance signal detection and transduction. ${ }^{62,63}$ Nanotechnology has also enabled advances in optical biosensor technology in the form of surface-enhanced Raman scattering (SERS). With SERS, one can achieve a degree of multiplicity more than current methods. SERS can measure up to 20 biomarkers at one time without any interference. ${ }^{64}$
The development of microfluidic laboratory on a chip (LOC) devices is a classic example of how nanotechnology can improve patient care. LOC technology takes the complexity of a laboratory and simplifies it into a low-cost, easy-to-use, portable system that can be used by physicians or patients. LOC methods that incorporate immunoassays and DNA hybridization arrays have been tested for their ability to identify individuals who may be at a high cancer risk. ${ }^{59}$ Another major application of nanotechnology is the use of quantum dots. Quantum dots are luminescent nanocrystals that have many of the same properties as optical biosensors. ${ }^{17}$ Quantum dots can emit light of different wavelengths, intensity, and spectral width, allowing for the diagnosis and detection of multiple unique molecular elements. ${ }^{58}$ They are able to track molecules and entire cells as they move through an environment. As such, they can be very important in monitoring cancer development by tracking the migration of cancer cells, cancer metastasis, and drug therapy effectiveness. The allure of quantum dots is their high stability, multimodality, and small size ( $\sim 50-100$ units in diameter for biological applications). Quantum dots are also capable of delivering therapeutic agents to specific target sites to improve pharmaceutical effectiveness while minimizing side effects. ${ }^{65}$ Similarly, the use of dendrimers as drug delivery systems and for effective targeting strategies has also emerged from the field of nanotechnology. ${ }^{66}$

\section{Conclusion}

Cancer biomarkers are important indicators of tumor growth. They are used not only to diagnose and monitor disease, but also to provide a prognostic approach to treatment. One of the ultimate goals of biosensor technology is POC cancer diagnosis. Point-of-care-testing (POCT), or diagnostic testing that is done on site, is a field in which biosensors could have a major impact, allowing the patient and medical staff to get results rapidly and easily. POCT allows for faster diagnosis and has the potential to significantly reduce costs. ${ }^{67}$ However, to move biosensors toward POC devices, multitarget detection of multiple biomarkers is necessary. In addition, POCT and multibiosensors, while bringing biosensor technology to the patient's bedside, must maintain the accuracy and reliability of the laboratory. ${ }^{47}$ LOC biosensors are a relatively new technology that show promise in this area. The major influences of nanotechnology on biosensor development involve nanomaterials, particularly quantum dots, as they cannot only facilitate diagnosis and tracking of cancer cells, but also can deliver drugs to target sites with precision and allow for more sensitive imaging systems that 
can detect cancer at an earlier stage. In the next 5-10 years, it is certain that nanotechnology will revolutionize cancer diagnosis and therapy. Integrating nanomaterials and biosensors will allow us to detect disease earlier, improve cancer imaging, and aid in diagnosis/prognosis and advance drug delivery while minimizing adverse reactions. Cancer takes place on the nanoscale level; it only makes sense that combating this disease also occurs at the nanoscale. Although the complexity and diversity of cancer has posed many challenges in the medical field, biosensor technology has the potential to provide fast, accurate results, while maintaining cost effectiveness.

\section{Disclosure}

The authors report no conflicts of interest in this work.

\section{References}

1. Rasooly A, Jacobson J. Development of biosensors for cancer clinical testing. Biosens Bioelectron. 2006;21(10):1851-1858.

2. $\mathrm{Hu} \mathrm{Y}$. BRCA1, hormone, and tissue-specific tumor suppression. Int J Biol Sci. 2009;5(1):20-27.

3. EMORY University. Additional tumor suppressors. 2008. Available from: http://www.cancerquest.org/index.cfm?page=781. Accessed Sep 242010.

4. Fabregat I. Dysregulation of apoptosis in hepatocellular carcinoma cells. World J Gastroenterol. 2009;15(5):513-520.

5. American Cancer Society. Oncogenes and tumor suppressor genes. 2005. Available from: http://www.cancer.org/docroot/ETO/content/ ETO_1_4x_oncogenes_and_tumor_suppressor_genes.asp. Accessed Sep 242010

6. Gown AM. Current issues in ER and HER2 testing by IHC in breast cancer. Mod Pathol. 2008;21 Suppl 2:S8-S15.

7. King MW. Growth factors. 2009. Available from: http://themedicalbio chemistrypage.org/growth-factors.html. Accessed Sep 242010.

8. Farrell WE, Clayton RN. Epigenetic change in pituitary tumorigenesis. Endocr Relat Cancer. 2003;10(2):323-330.

9. Stirzaker C, Millar DS, Paul CL, et al. Extensive DNA methylation spanning the Rb promoter in retinoblastoma tumors. Cancer Res. 1997; 57(11):2229-2237.

10. Greger V, Debus N, Lohmann D, Hopping W, Passarge E, Horsthemke B. Frequency and parental origin of hypermethylated RB1 alleles in retinoblastoma. Hum Genet. 1994;94(5):491-496.

11. Ford D, Easton DF, Stratton M, et al. Genetic heterogeneity and penetrance analysis of the BRCA1 and BRCA2 genes in breast cancer families. The Breast Cancer Linkage Consortium. Am J Hum Genet. 1998;62(3):676-689.

12. Miki Y, Swensen J, Shattuck-Eidens D, et al. A strong candidate for the breast and ovarian cancer susceptibility gene BRCA1. Science. 1994;266:66-71.

13. Falette N, Paperin MP, Treilleux I, et al. Prognostic value of P53 gene mutations in a large series of node-negative breast cancer patients. Cancer Res. 1998;58(7):1451-1455.

14. NCI. Biomarker. 2009. Available from: http://www.cancer.gov/diction ary/?searchTxt=biomarker. Accessed Sep 242010.

15. Basil CF, Zhao Y, Zavaglia K, et al. Common cancer biomarkers. Cancer Res. 2006;66(6):2953-2961.

16. Clinical practice guidelines for the use of tumor markers in breast and colorectal cancer. Adopted on 1996 May 17 by the American Society of Clinical Oncology. J Clin Oncol. 1996;14(10):2843-2877.

17. Tothill IE. Biosensors for cancer markers diagnosis. Semin Cell Dev Biol. 2009;20(1):55-62.
18. Smith DS, Humphrey PA, Catalona WJ. The early detection of prostate carcinoma with prostate specific antigen: the Washington University experience. Cancer. 1997;80(9):1852-1856.

19. Bill-Axelson A, Holmberg L, Ruutu M, et al. Radical prostatectomy versus watchful waiting in early prostate cancer. $N$ Engl J Med. 2005; 352(19): 1977-1984.

20. NCI. Prostate specific antigen (PSA) test. 2009. Available from: http:// www.cancer.gov/cancertopics/factsheet/detection/PSA. Accessed Sep 242010.

21. Thompson IM, Ankerst DP. Prostate-specific antigen in the early detection of prostate cancer. CMAJ. 2007;176(13):1853-1858.

22. Meyer T, Rustin GJ. Role of tumour markers in monitoring epithelial ovarian cancer. Br J Cancer. 2000;82(9):1535-1538.

23. Tuxen MK, Soletormos G, Dombernowsky P. Tumor markers in the management of patients with ovarian cancer. Cancer Treat Rev. 1995; 21(3):215-245.

24. Neesham D. Ovarian cancer screening. Aust Fam Physician. 2007; 36(3):126-128.

25. Kui Wong N, Easton RL, Panico M, et al. Characterization of the oligosaccharides associated with the human ovarian tumor marker CA 125. J Biol Chem. 2003;278(31):28619-28634.

26. Seelenmeyer C, Wegehingel S, Lechner J, Nickel W. The cancer antigen CA 125 represents a novel counter receptor for galectin-1. J Cell Sci. 2003;116(Pt 7):1305-1318.

27. Duffy MJ. Serum tumor markers in breast cancer: are they of clinical value? Clin Chem. 2006;52(3):345-351.

28. Tampellini M, Berruti A, Gerbino A, et al. Relationship between CA 15-3 serum levels and disease extent in predicting overall survival of breast cancer patients with newly diagnosed metastatic disease. Br J Cancer. 1997;75(5):698-702.

29. Bast RC Jr, Ravdin P, Hayes DF, et al. 2000 update of recommendations for the use of tumor markers in breast and colorectal cancer: clinical practice guidelines of the American Society of Clinical Oncology. J Clin Oncol. 2001;19(6):1865-1878.

30. Jager E, Stockert E, Zidianakis Z, et al. Humoral immune responses of cancer patients against "Cancer-Testis" antigen NY-ESO-1: correlation with clinical events. Int J Cancer. 1999;84(5):506-510.

31. Kubokawa M, Nakashima M, Yao T, et al. Aberrant intracellular localization of RCAS1 is associated with tumor progression of gastric cancer. Int J Oncol. 2001;19(4):695-700.

32. Dutsch-Wicherek M, Tomaszewska R, Lazar A, Wicherek L, Skladzien J. The association between RCAS1 expression in laryngeal and pharyngeal cancer and its healthy stroma with cancer relapse. BMC Cancer. 2009;9:35.

33. Alaoui-Jamali MA, Xu YJ. Proteomic technology for biomarker profiling in cancer: an update. J Zhejiang Univ Sci B. 2006;7(6):411-420.

34. Hanash SM, Pitteri SJ, Faca VM. Mining the plasma proteome for cancer biomarkers. Nature. 2008;452(7187):571-579.

35. Simpson KL, Whetton AD, Dive C. Quantitative mass spectrometrybased techniques for clinical use: biomarker identification and quantification. J Chromatogr B Analyt Technol Biomed Life Sci. 2009;877(13): $1240-1249$.

36. Wong SC, Chan CM, Ma BB, et al. Advanced proteomic technologies for cancer biomarker discovery. Expert Rev Proteomics. 2009;6(2): 123-134.

37. Chaplin M. What are biosensors? 2004. Available from: http://www. 1sbu.ac.uk/biology/enztech/biosensors.html. Accessed Sep 242010.

38. Chambers JP, Arulanandam BP, Matta LL, Weis A, Valdes JJ. Biosensor recognition elements. Curr Issues Mol Biol. 2008;10(1-2):1-12.

39. Hirsch LR, Jackson JB, Lee A, Halas NJ, West JL. A whole blood immunoassay using gold nanoshells. Anal Chem. 2003;75(10): 2377-2381.

40. Wu G, Ji H, Hansen K, et al. Origin of nanomechanical cantilever motion generated from biomolecular interactions. Proc Natl Acad Sci US A. 2001;98(4):1560-1564.

41. O'Connell PJ, Guilbault GG. Future trends in biosensor research. Anal Lett. 2001;34(7):1063-1078. 
42. Chen YT, Scanlan MJ, Sahin U, et al. A testicular antigen aberrantly expressed in human cancers detected by autologous antibody screening. Proc Natl Acad Sci U S A. 1997;94(5):1914-1918.

43. Jager D, Stockert E, Gure AO, et al. Identification of a tissue-specific putative transcription factor in breast tissue by serological screening of a breast cancer library. Cancer Res. 2001;61(5):2055-2061.

44. Parker S, Lim Y, Lee D, et al. Identification and characterisation of a novel cancer/testis antigen gene CAGE-1. Biochim Biophys Acta. 2003; 1625(2):173-182.

45. Tothill IE, Turner APF. Bionsensors. In: Caballero B, Trugo L, Finglas P, editors. Encyclopedia of Food Sciences and Nutrition. New York, NY: Academic Press; 2003:489-499.

46. Jia Y, Qin M, Zhang H, et al. Label-free biosensor: a novel phage-modified Light Addressable Potentiometric Sensor system for cancer cell monitoring. Biosens Bioelectron. 2007;22(12):3261-3266.

47. Wang J. Electrochemical biosensors: towards point-of-care cancer diagnostics. Biosens Bioelectron. 2006;21(10):1887-1892.

48. Mikkelsen SR. Electrochemical biosensors for DNA sequence detection. Electroanalysis. 1996;8(1):15-19.

49. Gooding JJ. Electrochemical sensors for DNA interactions and damage. Electroanalysis. 2002;14:1149-1156.

50. Wang J, Kawde A-N. Pencil-based renewable biosensor for label-free electrochemical detection of DNA hybridization. Anal Chim Acta. 2001; 431(2):219-224.

51. Koschwanez HE, Reichert WM. In vitro, in vivo and post explantation testing of glucose-detecting biosensors: current methods and recommendations. Biomaterials. 2007;28(25):3687-3703.

52. Tamada JA, Garg S, Jovanovic L, Pitzer KR, Fermi S, Potts RO. Noninvasive glucose monitoring: comprehensive clinical results. Cygnus Research Team. JAMA. 1999;282(19):1839-1844.

53. Asphahani F, Zhang M. Cellular impedance biosensors for drug screening and toxin detection. Analyst. 2007;132(9):835-841.

54. Chan LL, Gosangari SL, Watkin KL, Cunningham BT. A label-free photonic crystal biosensor imaging method for detection of cancer cell cytotoxicity and proliferation. Apoptosis. 2007;12:1061-1068.
55. Jacobson KB. Biosensors and other medical and environmental probes. Available from: http:/www.ornl.gov/info/ornlreview/rev29_3/text/ biosens.htm. Accessed Sep 242010.

56. Dell'Atti D, Tombelli S, Minunni M, Mascini M. Detection of clinically relevant point mutations by a novel piezoelectric biosensor. Biosens Bioelectron. 2006;21(10):1876-1879.

57. Medley CD, Smith JE, Tang Z, Wu Y, Bamrungsap S, Tan W. Gold nanoparticle-based colorimetric assay for the direct detection of cancerous cells. Anal Chem. 2008;80(4):1067-1072.

58. Grodzinski P, Silver M, Molnar LK. Nanotechnology for cancer diagnostics: promises and challenges. Expert Rev Mol Diagn. 2006; 6(3):307-318

59. Banerjee HN, Verma M. Use of nanotechnology for the development of novel cancer biomarkers. Expert Rev Mol Diagn. 2006;6(5):679-683.

60. Zhang GJ, Chua JH, Chee RE, Agarwal A, Wong SM. Label-free direct detection of MiRNAs with silicon nanowire biosensors. Biosens Bioelectron. 2009;24(8):2504-2508.

61. Wang J. Carbon-nanotube based electrochemical biosensors: a review. Electroanalysis. 2005;17:7-14.

62. Cai H, Cao X, Jiang Y, He P, Fang Y. Carbon nanotube-enhanced electrochemical DNA biosensor for DNA hybridization detection. Anal Bioanal Chem. 2003;375(2):287-293.

63. Yu X, Munge B, Patel V, et al. Carbon nanotube amplification strategies for highly sensitive immunodetection of cancer biomarkers. $J \mathrm{Am}$ Chem Soc. 2006;128(34):11199-11205.

64. Jain KK. Applications of nanobiotechnology in clinical diagnostics. Clin Chem. 2007;53(11):2002-2009.

65. Howarth M, Takao K, Hayashi Y, Ting AY. Targeting quantum dots to surface proteins in living cells with biotin ligase. Proc Natl Acad Sci US A. 2005;102(21):7583-7588.

66. Bharali DJ, Khalil M, Gurbuz M, Simone TM, Mousa SA. Nanoparticles and cancer therapy: a concise review with emphasis on dendrimers. Int J Nanomedicine. 2009;4:1-7.

67. Moving biosensors to point-of-care cancer diagnostics. Proceedings of a symposium. 2005 Jun. Biosens Bioelectron. 2006;21(10):1847-1942.
Nanotechnology, Science and Applications

\section{Publish your work in this journal}

Nanotechnology, Science and Applications is an international, peerreviewed, open access journal that focuses on the science of nanotechnology in a wide range of industrial and academic applications. It is characterized by the rapid reporting across all sectors, including engineering, optics, bio-medicine, cosmetics, textiles, resource sustainability

\section{Dovepress}

and science. Applied research into nano-materials, particles, nanostructures and fabrication, diagnostics and analytics, drug delivery and toxicology constitute the primary direction of the journal. The manuscript management system is completely online and includes a very quick and fair peer-review system, which is all easy to use. 